\title{
Addressing Mental Health in Planetary Science
}

A White Paper for the NRC Planetary Science and Astrobiology Decadal Survey

This White Paper is submitted as part of a collaborative effort organized by the Equity, Diversity, and Inclusion Working Group (EDIWG), a cross Assessment Group (AG) committee.

Authors: S. D. Vance (he/him/his) ${ }^{1}$, C. Elder ${ }^{1}$, A. Hofmann ${ }^{1}$, S. Howell ${ }^{1}$, M. Milazzo (he/him/his) ${ }^{2}$, R. T. Pappalardo ${ }^{1}$, J. Noviello $^{3}$, D. A. Patthoff ${ }^{4}$, Z. Khan ${ }^{5}$, J. Rathbun ${ }^{4}$, J. Vertesi ${ }^{6}$

${ }^{1}$ Jet Propulsion Laboratory, California Institute of Technology, Pasadena, CA (626-437-6200; svance@jpl.caltech.edu)

${ }^{2}$ Other Orb LLC, Flagstaff, AZ

${ }^{3}$ School of Earth and Space Exploration, Arizona State University, Tempe, AZ

${ }^{4}$ Planetary Science Institute, Tucson, AZ

${ }^{5}$ Unafilliated, Pasadena, CA

${ }^{6}$ Princeton University, Princeton, NJ

The list of signatories may be found here. If you wish to be added to the list please use this form. 
Acknowledgements: A portion of this research was carried out at the Jet Propulsion Laboratory, California Institute of Technology, under a contract with the National Aeronautics and Space Administration. (C) 2020.

\section{Introduction}

As NASA strives to be more inclusive by confronting institutional racism, gender inequality, and sexual harassment, and by recognizing sexual and gender diversity, it must also work to address a compounding crisis of mental health [1]. Even before the global pandemic, concerns were being raised about the costs to mental well-being of the demanding academic environment. A parallel problem identified in the field of psychology highlights how expectations in American culture particularly place excessive burdens on the individual:

"In many ways, academia mirrors other institutions of power and privilege that are based on race, gender, social class, and other socially constructed categories... The endorsement in U.S. society of rugged individualism and the illusion of meritocracy have been reinforced in the education and training in psychology that have had a strong bias toward framing human problems in 'apolitical, intrapsychic, and deficit-oriented diagnoses." [1].

Though there is clear evidence that the demands of an academic career create many potential triggers for psychological distress, it remains difficult to gauge the extent to which these problems affect US science in general and planetary science in particular. From a recent study by the RAND Corporation, a nonprofit institution providing research and analyses to policymakers:

"More work is needed to understand both the mental health needs of researchers and how they can be addressed [including the] effectiveness of interventions, prevalence of specific mental health needs (rather than stress) among researchers, and any evidence about researchers outside the academic setting. There are also limitations to the quality and design of many of the studies conducted, such as lack of long-term follow-up and absence of control groups." [2].

Here, we summarize the available evidence for a mental health crisis among academics. We describe how this problem intersects with and amplifies problems of equity, diversity, and inclusion. Addressing the problems is not just a matter of doing the right thing, it also holds the promise of creating a more creative and effective workforce.

\section{This paper's main finding is that NASA should invest in efforts to understand the scope and impact of mental health problems in its scientific workforce. NASA should address such challenges among its scientific workforce by funding intervention strategies, providing incentives to reward effective mentorship, fostering healthier work habits, and equitably supporting non-traditional work styles that may suit neurodiverse individuals.}


In our language and description of symptoms described we have strived for consistency with the Diagnostic and Statistical Manual of Mental Disorders, 5th edition [3], but we emphasize that none of the authors of this paper has formal training in psychology or psychiatry.

Evidence for a mental health crisis among academic researchers: Recent studies that reveal a high prevalence of anxiety and depression among a diverse sample of graduate students [4] point to a need for more comprehensive career development programs across institutions, to support awareness and management of mental health. Institutions providing such interventions may have competitive advantages over those that do not by attracting and retaining students, postdoctoral researchers, and faculty. The authors advocate for training programs pairing mental health professionals with faculty, administration, and department staff. These impacts do not stop at graduate school, given the compounding pressures of establishing a career [5]. Numerous blog posts by academics ${ }^{1,2,3}$ in recent years have highlighted the emotional toll of balancing the demands of personal life while building a research program and seeking tenure. In the recent survey of the planetary workforce [6], the single most important item that negatively affects respondent's careers is the balance between their work and personal life.

Mental health as a cross-cutting theme: Causes and triggers of poor mental health are not fully understood, but risk factors include impoverished and traumatic upbringings, and membership in groups labeled as underrepresented. The intersectional nature of the problem is illustrated by the list of subtopics in a recent call for papers ${ }^{4}$ for a book on mental health in academia:

- general trends in mental health issues in academia

- systematic/structural contributors [...] workload/ tenure expectations, gender[...]

- comparative [...] mental health issues in academia in the United Studies and other countries

- workplace bullying and mental health in academia

- critical examinations of the stigma and silence around mental health in academia

- relationships between mental health and LGBTQ identity

- intersections between mental health issues and race/ethnicity

- managing mental health as a chronic illness

- intersections between mental health and gender

- mental health and class in the academy

- mental health for employees with job insecurity (such as adjunct positions)

1 Essay on dealing with depression while on the tenure track

2Guest Post: Research, Teaching, Service...and Depression | Tenure, She Wrote

3 Essay on avoiding depression after being awarded tenure

4 Coming Soon in 2021: Mental Health Among Higher Education Faculty, Administrators, and

Graduate Students: A Critical Perspective 
- relationships between emotional labor and mental health in academia

- mental health, stress, and burnout in academia

- mental health as a diversity, equity, and/or political issue in academia

- mental health issues among graduate students, faculty, staff, and administrators who were first in their families to attend college

- adjustment to academia and campus life for graduate students, faculty, and administrators with mental health issues

- research on ways academics can support and improve mental health

- $[\ldots]$ developing institutional initiatives that address mental health, mental health literacy, and related issues (work-life balance, productivity, general wellness, and other related topics)

The extraordinary stress of academic life as a trigger for depression, anxiety, and burnout should not cause us to ignore other types of mental illness. The incidence of other types of mental illness such as personality disorders and psychoses together affect about $5 \%$ of the adult population, but little is known about the prevalence or impact of these illnesses among academics [5]. At least one recent study suggests a higher prevalence of schizophrenia and bipolar among people in academia [7]. Here we provide a sampling of conditions among individuals and available information on their roles in academic life. These vignettes also illustrate the hoped-for benefits of cultivating an academic environment that is more supportive of mental health.

Schizophrenia: The story of Elyn Saks ${ }^{5,6}$, a USC professor, demonstrates the possibility for some people living openly with schizophrenia to thrive in academia. Her experience being fired from an earlier position on grounds of negligence for not disclosing the condition also underscores the need for creating a culture where mental illness is not stigmatized, and where people are trained to spot signs of mental health crisis and to be supportive.

Anxiety Disorders include generalized anxiety (described above), panic, and obsessive compulsive. We focus here on Obsessive Compulsive (OCD). The international OCD Foundation describes obsessive compulsive disorder as follows": "In order for a diagnosis of obsessive compulsive disorder to be made, [a] cycle of obsessions and compulsions becomes so extreme that it consumes a lot of time and gets in the way of important activities that the person values....Common obsession themes: contamination, religious obsessions (scrupulosity), fear of causing harm, symmetry/perfectionism, unwanted sexual thoughts". Manifestations will vary by the particular theme. Many obsessions may include avoiding certain situations or locations.

Mood Disorders include depression, bipolar, and borderline personality.

${ }^{5}$ Why schizophrenia need not rob us of a life in academia

6 A tale of mental illness -- from the inside

? What is OCD? 
Depression is described as involving persistent feelings of sadness and loss of interest. It can have numerous symptoms that may not be subjectively described as sadness ${ }^{8}$, including feelings of hopelessness, guilt, shame, worthlessness, and a loss of interest in previously enjoyed things and activities. Some people may become more impulsive and aggressive during intense bouts of depression. Physical symptoms manifest as changes to eating, exercise, sleeping, and socialization routines (i.e., doing much more or much less of these activities) or unusual bodily pain such as headaches, and often exacerbate psychological symptoms. Episodes of depression can be triggered by life events ${ }^{9,10}$, large or small, and may last only a few days, or much longer. A recent article in Science Magazine recommends:

"The entire academic community needs to tackle the systemic factors that likely
contribute to mental distress. Institutions should implement adequate support systems
for mental health. Principal investigators should lead by example in promoting their own
well-being and the well-being of their trainees."

People with depression may not seek help because they see their problems as burdensome or not important enough to talk about. Supervisors and peers should encourage them to seek help.

Bipolar disorder (f.k.a. manic-depressive disorder) comes in two primary flavors: Bipolar I and Bipolar II. Although each involves clear cyclic changes in mood, energy, and activity level-from periods in which an individual is extremely "up" or "high" (manic or hypomanic episodes) to periods in which an individual is extremely "down" (depressive episodes) and periods in which symptoms of both episodes are mixed together-the duration of these cycles (i.e., how frequently an individual cycles from high to low and back again), the duration of each high or low period itself, and the severity of the highs and lows differ.

Like Elyn Saks, Kay Redfield Jamison, Co-Director of the Mood Disorders Center and Professor of Psychiatry and Behavioral Sciences at the Johns Hopkins University School of Medicine, has spoken and written openly and frankly about her life-long struggle with and embrace of bipolar disorder. In her book An Unquiet Mind: A Memoir of Moods and Madness, Jamison details the literal highs and darkest lows of her experiences with bipolar disorder.

Individuals with bipolar disorder benefit from flexible schedules. Some use hypomanic periods to accomplish higher work output, and may choose to not treat symptoms due to positive feedback from these periods. Regular sleep cycles are essential for symptom maintenance, as work schedules not providing for adequate rest may trigger bipolar episodes. Scientists with collaborators who identify as bipolar should take extra care in setting work schedules.

$\stackrel{8}{ }$ Depression (major depressive disorder) - Symptoms and causes

9 -The Relationship of Childhood Abuse to Impulsivity and Suicidal Behavior in Adults With Major Depression

${ }^{10}$ Postpartum depression, suicidality, and mother-infant interactions

${ }^{11}$ Why World Mental Health Day matters for scientists 


\section{Moreover, collaborators should be considerate of colleagues who might need a specific work schedule for undisclosed reasons.}

Borderline Personality: Students living with the borderline personality disorder may need to terminate an otherwise successful term of study. One article on the topic notes the importance for such students to have a supportive therapist and plan for the term in order to anticipate and work through upsetting events ${ }^{9}$. Improving the academic setting to be more supportive of mental health would reinforce such measures.

Substance Use Disorders have a strong co-occurrence with mood disorders [8]. The stresses of academic life provide numerous triggers for addictive behavior, which shares compulsive aspects but is distinct for involving pleasure-seeking behavior and denial of associated harm ${ }^{10}$. The cultures of many academic disciplines can also encourage heavy drinking, as detailed in a recent article in Science Magazine ${ }^{11}$. In such environments, admitting to having trouble controlling one's drinking can be seen as a moral failure or professional lapse. Niki Kiepek, who studies substance abuse, suggests that organizations should gather anonymized data on substance use and share it with members. "For those who fear they have problems with their use, just knowing a certain percentage of fellow professionals also report problems 'normalizes it a bit, or it contextualizes it,' she says, 'so then that person doesn't feel alone."' Recent prohibitions against serving alcohol at conference poster and oral sessions at some geoscience meetings may be a first step to create more inclusive settings for non-drinkers and for those abstaining ${ }^{12}$.

Autism Spectrum Disorder (ASD) is a group of developmental disabilities characterized by challenges with social interaction and communication. People with ASD typically depend on structured routine, may exhibit self-soothing physical actions such as rocking and fidgeting, have difficulty initiating and maintaining social relationships, and/or have trouble with interpersonal communication. People with ASD will require different levels of support throughout their education and employment. Because ASD symptoms vary in severity between individuals, people with ASD have been referred to as "high-" or "low-functioning." The ASD community prefers the terms "high-" and "low-support" to emphasize an institution's ADA-based responsibility to provide equitable treatment to students and employees. Many people with ASD may have difficulties finding jobs matching their skill and education levels, or will struggle during interviews or when building their resumes. People with ASD are also more likely than a neurotypical person to experience depression [9] and suicidal ideation [10]. It is largely through the perception of material support from others that adults with ASD feel more comfortable in

${ }^{2}$ Borderline Personality Disorder and College Success

10 The Difference Between an Addiction and a Compulsion

11 In some disciplines, heavy drinking is part of the culture. That can be a problem

12 GSA News Release 20-20 
their jobs. Parents who have children with ASD are also impacted. Children with ASD often require more attention from their parents, which can impact the parents' employment.

Reducing stigma around mental illness: Social stigma is one major component of why individuals with mental illness do not seek treatment from professionals or understanding from their peers. The disclosure of mental illnesses often leads to negative interactions with one's peers, friends, family, employers and mentors, etc. A substantial reduction of stress associated with mental illness thus could be accomplished by reducing stigmas. A meta-analysis of national and global efforts to accomplish stigma and discrimination reduction [SDR; 11], identified key areas where more research is needed before recommendations could be produced:

The effects of SDR programs on a broader set of outcomes: Research is needed to understand whether SDR programs encourage nondiscriminatory policy or behavior, and whether SDR programs increase treatment-seeking among people with symptoms

The effects of broad-based SDR programs: Research is needed to understand the effects of broad-based SDR programs that combine education and training among members of the U.S. population, using rigorous methods that involve comparison groups

Follow-up of SDR program participants: more research is needed on the longevity of attitude changes after an SDR campaign or training has ended. Attitudes shift after such interventions, but little is known about whether these changes persist weeks or months later

Another stigma that is often overshadowed by public or peer-based perceived stigma (stereotype awareness or stereotype threat) is self-stigma, in which an individual internalizes stigma through stereotype agreement, self-concurrence, and self-esteem adjustment [12,13]. Because of this self-stigma, an individual may fear seeking help even in a supportive environment. Both education and contact with individuals with mental illness can reduce stigma [12].

Efforts to improve mental health in planetary science are underway, even in the absence of the agency-wide demographic surveys and workforce improvements recommended here. Institutions and funding agencies should encourage such grassroots efforts.

For example, the Department of Chemistry of the University of Minnesota recently partnered with Boynton Health to survey the mental health of its graduate student population [14]. Conducted in 2013 and 2016, the anonymous survey included questions about "demographics; funding situation; progress toward degree; work hours; physical health (sleep patterns, use of alcohol and recreational drugs, exercising habits), mental health (social isolation and depression symptoms); relationship with the academic advisor, group members, and other members of the department; stress and anxiety in relation to different factors; and help-seeking behaviors when facing mental health issues." Participation was high: $\sim 50 \%$. As a result of the surveys, the departmental culture shifted to more candidly talk about stress and mental health. Faculty 
seminars began including advice on managing mental health, and the graduate students organized a digital library containing advice on "Surviving and Thriving in Graduate School".

Recommended Accommodations: Several cross-cutting interventions are identified above. The following accommodations will help to provide a more equitable work environment in general:

Foster a culture that supports healthy sleep habits, e.g., "sleep is for the strong"13 Encourage flexible work arrangements, including work-from-home options Design supportive scheduling protocols, working with mental health experts, that provide recovery buffers for people with mental illnesses

Encourage supportive conference planning, with scheduled breaks for daily rest. This includes planning for social and networking events to occur earlier in the day Encourage supportive conference social events that don't serve alcohol Establish protocols for clear communication. Severe anticipatory anxiety can arise from vague emails, e.g. from a supervisor or HR stating "I need to talk to you about something" Many people may experience symptoms severe enough to be considered a disability ${ }^{14}$. Accommodations vary for individuals. Employers should note that psychiatric illnesses affect many body systems, and should avoid treating them as "stress" dissimilar to other illnesses.

Time off: Clear procedures for requesting time away, including the ability to designate a person - e.g., next of kin or a partner - to communicate on a patient's behalf. Many psychiatric facilities do not allow patients to bring electronics with them, so being able to delegate communication can be essential for completing paperwork. Given the exceptional work expected of researchers, institutions should consider offering longer sabbatical terms, and non-traditional research organizations should consider paid leave, e.g. 6-12 months for every 5-7 years worked.

Disability insurance: Organizations should consider offering short-term benefits beyond the typical $60 \%$ level, as disability leave often results in higher living and medical expenses, and loss of income is a major stressor affecting recovery. Some organizations limit long-term disability leave benefits for psychiatric illnesses. This is a form of discrimination arising from the social stigma and lack of understanding of psychiatric illnesses.

References: [1] Shin, R.Q. (2008) J. Multicultural Counseling Dev. 36, 180-191. [2] Guthrie, S. et al. (2017) RAND Corporation RR-20222-RS, doi.org/10.7249/RR2022 [3] American Psychiatric Association. (2013). American Psychiatric Pub. [4] Evans, T.M. et al. (2018) Nature Biotechnology, 282-284 [5] Guthrie, S. et al.(2018). RAND health quarterly, 7(3) [6] Hendrix et al. (2020) LPSC Abstract \# 2813. [7] Parnas, J. et al. (2019). Frontiers in psychiatry, 10, 175 [8] Quello, S.B. et al. (2005). Science \& Practice Perspectives, 3(1), 13 [9] Hofvander, B., et al. (2009) BMC Psychiatry 9, 1-9. [10] Segers, M., \& Rawana, J. (2014) Autism Research 7,

$\underline{13}$ Sleep is for the Strong

14 Mental Health Impairments 
507-521 [11] Corrigan, et al. (2006). J. Soc. Clinical Psych., 25(8), 875-884 [12] Corrigan, P. W. et al. (2012). Psychiatric services, 63(10), 963-973 [13] Schmitt, M. T. et al. (2014).

Psychological bulletin, 140(4), 921 [14] Mousavi, M. P. et al. (2018). Journal of Chemical Education, 95(11), 1939-1946. 